\title{
OUTROS DISTÚRBIOS DO SONO NA SÍNDROME DA APNÉIA DO SONO
}

\author{
SLEEP DISORDERS ASSOCIATED WITH SLEEP APNEA SYNDROME
}

\author{
Heidi H Sander ${ }^{1}$, Daniela V Pachito², Letícia S Vianna ${ }^{3}$
}

\begin{abstract}
${ }^{1}$ Médica Assistente 2.Pós-graduanda. ${ }^{3}$ Médica Colaboradora. Departamento de Neurologia Psiquiatria e Psicologia Medica Faculdade de Medicina de Ribeirão Preto - USP.

CORRESPONDÊNCIA: Heidi H Sander.

Departamento de Neurologia, Psiquiatria e Psicologia Médica. Faculdade de Medicina de Ribeirão Preto- USP.

Av. Bandeirantes 3900, Monte Alegre. CEP 14049-900, Ribeirão Preto- SP, Brasil.

E-mail: birrdy@netsite.com.br
\end{abstract}

Sander HH, Pachito DV, Vianna LS. Outros distúrbios do sono na Síndrome da Apnéia do Sono. Medicina (Ribeirão Preto) 2006; 39 (2): 205-211.

RESUMO: Vários distúrbios do sono podem acompanhar a Síndrome da Apnéia do Sono pela concomitância das síndromes ou pela existência de mecanismos fisiopatológicos comuns. Destacamos a insônia, o bruxismo do sono e o sonambulismo.

A insônia pode ser uma das queixas dos pacientes portadores de distúrbios respiratórios do sono, pela fragmentação do sono decorrente. Além disso, ambos podem trazer sérias repercussões na saúde como o aumento da morbidade cardiovascular e maior risco de acidentes automobilísticos e laborativos, como tem sido demonstrado nas duas ultimas décadas. O correto diagnóstico de insônia em pacientes com apnéia do sono, bem como de apnéia em pacientes com insônia define a metodologia propedêutica empregada e também o tratamento, como por exemplo, a abordagem farmacológica quando necessária e o tratamento do distúrbio respiratório concomitantemente.

O bruxismo do sono pode estar presente em $3,5-14 \%$ dos pacientes com apnéia do sono. $\mathrm{Na}$ sua investigação clínica devemos sempre avaliar a possível presença da apnéia do sono já que para o seu tratamento muitas vezes é necessária a sua abordagem em primeiro lugar para então utilizarmos tratamentos farmacológicos e dispositivos que diminuam os danos às estruturas orofaciais.

Dentre as parassonias que frequentemente acompanham a apnéia do sono temos o sonambulismo. Ele representa a presença de atividade motora típica da vigília durante estado do sono. Esse fenômeno torna-se mais freqüente quando ocorre fragmentação do sono, provocando dissociação das variáveis fisiológicas, como ocorre em indivíduos com distúrbios respiratórios do sono.

Concluindo, é de suma importância o conhecimento e a identificação de outros distúrbios do sono que podem ocorrer como epifenômenos ou como conseqüência da apnéia obstrutiva do sono, pois tanto o tratamento dos mesmos pode, em alguns casos agravar a apnéia do sono, quanto o subdiagnóstico pode interferir na melhora clínica do paciente.

Descritores: Insônia. Bruxismo do Sono. Sonambulismo. Síndromes da Apnéia do Sono. 
Dentre os distúrbios do sono que freqüentemente acompanham os distúrbios respiratórios obstrutivos (DROS), quer pela concomitância das síndromes, quer por ser desencadeados pelos mesmos mecanismos fisiopatológicos encontram-se a insônia, o bruxismo do sono e as parassonias.

\section{1- INSÔNIA}

\section{1- Considerações gerais}

A Insônia e a Síndrome da Apnéia Hipopnéia Obstrutiva do Sono (SAHOS) são distúrbios do sono freqüentes, que levam a fragmentação do sono e a uma série de repercussões na saúde como o aumento da morbidade cardiovascular e um maior risco de acidentes (WHO, 2004) ${ }^{1}$. A maioria dos estudos prévios investiga cada uma destas síndromes e seus efeitos isoladamente. Os pacientes com Síndrome da Apnéia Hipopnéia Obstrutiva do Sono não costumam referir uma dificuldade para iniciar o sono. Por outro lado, os pacientes com Insônia não são comumente investigados quanto a possíveis alterações respiratórias. Somente nas duas últimas décadas é que surgiram artigos aventando tanto a possibilidade de um diagnóstico oculto de Síndrome da Apnéia Hipopnéia Obstrutiva do Sono (SAHOS) em pacientes com Insônia, quanto outros estudos reportando uma maior ocorrência de Insônia em pacientes com SAHOS ${ }^{2,3,4}$. Em 1999, Lichstein et al. ${ }^{2}$ descreveram a ocorrência de SAHOS entre $29 \%$ a $43 \%$ de pacientes com Insônia. Complementando este achado, em 2001, Krakow et al. ${ }^{3}$ avaliaram retrospectivamente 231 pacientes com diagnóstico de SAHOS e encontraram até 50\% desta amostra com Insônia. Ao comparar o grupo com Insônia e SAHOS com o grupo que apresentava somente SAHOS, embora o índice de apnéia e hipopnéia (IAH) entre os grupos fosse similar, observou-se que o grupo com Insônia e SAHOS apresentava maior latência ao sono, reduzida eficiência de sono, maior frequiência de distúrbios psiquiátricos e sintomas cognitivos emocionais, menor qualidade de vida e maior uso de sedativos ou de hipnóticos ${ }^{4}$. Similarmente, em 2004, Smith et al. ${ }^{5}$ verificaram uma alta prevalência de Insônia em pacientes com síndrome da apnéia hipopnéia obstrutiva do sono. Estes autores observaram uma forte correlação entre Insônia e SAHOS, e detectaram uma maior freqüência de depressão, ansiedade e stress nos indivíduos que conjugavam estes dois distúrbios ${ }^{5}$. Recentemente, Krell e Kapur ${ }^{6}$ avaliaram retrospectiva- mente os dados de polissonografia de 255 pacientes com SAHOS e verificaram 54,9\% de indivíduos com queixas de Insônia (33.4\% déficit de iniciar o sono, $38.8 \%$ déficit para manter o sono; $31.4 \%$ despertares precoces). Interessante notar que a Insônia foi observada mais comumente em pacientes com SAHOS leve $(81.5 \%)$ em detrimento daqueles com grau moderado a grave $(51.86 \%)$. Krell e Kapur ${ }^{6}$ descreveram que os achados polissonográficos associados à Insônia foram IAH baixo e o índice de dessaturação pequeno. No subgrupo com SAHOS moderada a grave não houve associação entre Insônia versus IAH ou índice de dessaturação ${ }^{6}$. Estes mesmos autores ressaltaram diversos fatores associados ao grupo com Insônia e SAHOS, quais foram: o gênero feminino, a doença psiquiátrica, a dor crônica e a síndrome das pernas inquietas ${ }^{6}$.

\section{2- Diagnóstico}

Embora existam evidências indicando uma freqüente associação entre Insônia e SAHOS, ainda há uma necessidade de estudos prospectivos e multicêntricos avaliando não só a prevalência como também as implicações diagnósticas e terapêuticas desta associação. A diferença na metodologia empregada para investigação de Insônia e dos SAHOS leva a uma dificuldade diagnóstica inicial. Considerando o diagnóstico de SAHOS, a força tarefa empreendida pela Academia Americana de Medicina do Sono ${ }^{7}$, em 1999 determinou critérios clínicos e polissonográficos para o diagnóstico de certeza de um SAHOS. O critério clínico proposto foi a sonolência excessiva diurna não explicada por outras causas e mais que dois dos seguintes sintomas: engasgos durante o sono, despertares freqüientes, sono não restaurador, fadiga diurna e dificuldade de concentração (sem outra causa aparente). $\mathrm{O}$ critério polissonográfico estabelecido foi a presença de cinco ou mais episódios obstrutivos (apnéia, hipopnéia ou episódios de despertar relacionado a esforço respiratório) por hora de sono ${ }^{7}$. Já em relação à Insônia, o consenso para a investigação de Insônia foi o de que se exclua: distúrbios neurológicos, ambientais, de ritmo circadiano e distúrbio comportamental; doenças psiquiátricas; condições clínicas; distúrbio específico do sono como a síndrome das pernas inquietas e a Síndrome da Apnéia Obstrutiva do Sono, mas ao mesmo tempo não houve indicação de polissonografia ${ }^{8}$. Em resumo, os Distúrbios Respiratórios do Sono (como a SAHOS) entram no diagnóstico diferencial da Insônia, mas para o diagnóstico de certe- 
za dos SAHOS inclui-se não só a avaliação clínica, mas também a polissonografia; já para o diagnóstico de Insônia somente a avaliação clínica é tida como essencial. Como a polissonografia não é uma indicação absoluta na suspeita de Insônia, o diagnóstico de SAHOS nos pacientes com Insônia pode continuar oculto.

\section{3- Aspectos terapêuticos}

Além da metodologia diagnóstica um outro aspecto importante a ser abordado é a forma de tratamento diante da associação entre Insônia e SAHOS. Em relação à Insônia, até o momento, o tratamento mais freqüentemente utilizado tem sido a abordagem farmacológica associada ou não a terapia cognitiva/ comportamental ${ }^{9}$. Sabe-se que as medicações usadas para Insônia podem gerar efeitos adversos em indivíduos com distúrbios respiratórios como a SAHOS 9 . Em adição, embora o uso de hipnóticos e antidepressivos tenha sido amplamente difundido, seus efeitos a longo prazo ainda estão sendo investigados e são necessárias mais pesquisas para esclarecimentos ${ }^{10}$. Quanto a terapia cognitiva/comportamental, esta abordagem terapêutica vem sendo reconhecida como importante opção de tratamento, por ser efetiva e muitas vezes mais bem tolerada pelos pacientes ${ }^{11}$.

Finalmente, a detecção da maior ocorrência de Insônia nos pacientes com SAHOS levanta uma séria questão sobre o quanto melhor estes pacientes poderiam estar sendo diagnosticados e tratados a partir da verificação da sobreposição destas duas síndromes.

\section{2- BRUXISMO DO SONO}

\section{1- Considerações gerais}

O bruxismo do sono (BS) é categorizado de acordo com a Classificação Internacional dos Distúrbios do Sono proposta pela Academia Americana de Medicina do Sono ${ }^{12}$ como uma parassonia, isto é, um distúrbio que se sobrepõe ao processo de sono sem no entanto ser um distúrbio do sono per se. Chamamos de BS ao movimento rítmico, estereotipado de apertar ou ranger dos dentes que ocorre durante o sono. A contração da musculatura têmporo-mandibular que o produz pode ser tônica, no primeiro caso ou fásica, no segundo. Sabemos que a prevalência do BS é de 8090\% da população, com declínio linear conforme a idade de $19 \%$-entre 3-10 anos - para $13 \%$ no adulto jovem e $3 \%$ na população com mais de $60 \operatorname{anos}^{13,14}$.
Porém em apenas 5\% dos casos ele se apresenta como uma condição clínica. Não há diferença da prevalência entre os sexos e o BS geralmente se inicia após a troca da dentição decídua, ao final da primeira década de vida.

De acordo com a sua duração pode ser classificado como agudo, quando ocorre em menos de sete dias; subagudo, quando dura entre oito e trinta dias e crônico quando sua duração é superior a um mês. Ainda, em relação a sua gravidade, é considerado leve quando não está presente todas as noites, não causa danos à dentição ou danos psicossociais; moderado, quando ocorre todas as noites e causa danos à dentição ou psicossociais leves; e grave quando ocorre todas as noite e os danos para a dentição, articulação têmporo-mandibular ou psicológicos estão presentes e considerados moderados ou graves ${ }^{12,13}$.

\section{2- Clínica e fisiopatologia}

Os critérios diagnósticos propostos ${ }^{12}$ para o BS são:

1- queixa clínica de ranger ou apertar dos dentes durante o sono;

2- desconforto na musculatura maxilo-mandibular, presença de sons associados ao bruxismo, mudança ou dano aos dentes.

Dentre as complicações mais freqüentemente encontradas nos pacientes com BS temos as alterações da morfologia dos dentes, danos às estruturas periodontais tais como a retração ou inflamação da gengiva e a reabsorção do osso alveolar; hipertrofia da musculatura da mastigação e desordens da articulação têmporo-mandibular. A dor facial ou cefaléia temporal estão presentes nos casos mais graves e crônicos.

A associação entre BS e a Síndrome das Apnéias/Hipopnéias Obstrutivas do Sono (SAHOS) de grau leve a moderado pode ocorrer em até $50 \%$ dos pacientes ${ }^{15}$. Porém, conforme demonstrado por Ayappa et al. ${ }^{16}$, em somente $3,5 \%$ dos pacientes com SAHOS de grau leve e em $14,4 \%$ dos casos de SAHOS moderada os episódios de bruxismo ocorrem ao término do evento respiratório obstrutivo. Desta forma é postulado que, apesar do BS estar relacionado à SAHOS, não parece que os episódios de bruxismo estejam diretamente associados aos eventos respiratórios ${ }^{13,17,18}$.

Os mecanismos fisiopatológicos envolvidos na gênese do BS são ainda desconhecidos, sendo considerados multifatoriais: influência da atividade do sistema nervoso central (SNC) tais como oromotoras, 
da regulação do ciclo sono-vigília, autonômicas, catecolaminérgicas, genéticas e psicossociais. A presença de ativação eletrencefalográfica e cárdio-autonômica sugerem que o BS é conseqüência dos microdesper$\operatorname{tares}^{13}$.

\section{3- Avaliação diagnóstica}

O diagnóstico do BS baseia-se principalmente na anamnese e exame físico do paciente, sendo raramente necessária a realização de exames complementares, exceto nos casos em que haja suspeita da associação com Síndrome da Apnéia Obstrutiva do Sono, sendo então indicada a polissonografia.

A anamnese deve contemplar a pesquisa da presença de sons produzidos pelo ranger ou apertar dos dentes, dor ou desconforto facial matinal, cefaléia, sensibilidade dos dentes a alimentos frios ou quentes, a presença de fratura dentária ou de restaurações. O desgaste dos dentes, retração gengival, hipertrofia da musculatura mastigatória, click à palpação da articulação têmporo-mandibular, podem estar presentes ao exame físico, especialmente nos casos mais avançados.

Os achados polissonográficos encontrados nos pacientes com BS são: atividade rítmica ou tônica dos músculos masseteres ou temporais durante o sono podendo ocorrer em qualquer estágio, sendo mais comum nos estágios I e II do sono não-REM (NREM). A arquitetura do sono geralmente é normal, porém muitas vezes há aumento dos microdespertares, do número de mudanças de estágios do sono e da freqüência cardíaca ${ }^{12,13}$.

Os critérios polissonográficos para o diagnóstico de BS consistem em:

a) aumento do tônus masseter $>10 \%$ ou $20 \%$ do máximo da calibração biológica (é solicitado ao paciente o apertar máximo dos dentes durante a vigília);

b) bruxismo fásico: presença de 3 ou mais surtos de aumento do tônus, com duração de 0,25-2 seg cada;

c) bruxismo tônico: aumento do tônus sustentado por mais de 2 seg;

d) bruxismo misto: associação dos dois tipos anteriores.

A quantificação do bruxismo é considerada anormal quando a frequiência do bruxismo tônico é $>4 /$ h de sono e fásico é $>25$ surtos $/$ h sono ${ }^{19}$.

\section{4- Tratamento}

O tratamento para o BS tem como principal objetivo diminuir os danos às estruturas orofaciais.
Apesar de ainda não estar comprovada a relação de causalidade entre os DROS e o SB, existe o consenso em se tratar primeiro o DROS, caso este esteja presente e, se se mantiver o BS, deverão ser adotadas outras formas de tratamento. Assim, temos a seguir as principais formas de tratamento paliativas para o $\mathrm{BS}^{19}$ :

Tabela I: Tratamento paliativo do Bruxismo do Sono.

\section{COMPORTAMENTAIS}

- Biofeedback

- Psicoterapia

- Terapia e Treinamento Cognitivo-Comportamental (relaxamento, respiração)

- Mudanças de estilo de vida que diminuam estresse

ORODENTAIS

- Placa miorrelaxante

- Tratamento oclusal

FARMACOLÓGICO

- Relaxantes musculares: buspirona (+),ciclobenzaprina (?)

- Inibidores seletivos da recaptação de serotonina: venlafaxina (+), trazodona (?)

- Agonistas dopaminérgicos: L-Dopa (+), pramipexol (?), pergolide (?), gama-hidroxibutirato $(+)$

- OUTROS: propranolol (+/?), clonidina (+) toxina botulínica (?)

\section{OUTRAS MODALIDADES}

- Tratamento da xerostomia: evitar cafeinados, álcool, nicotina; uso de sprays lubrificantes que mimetizem a saliva, drogas sialagogas (?)

Legenda: (+) eficácia comprovada, (?) eficácia questionada

\section{3- PARASSONIAS}

As parassonias são definidas como comportamentos indesejáveis ou desagradáveis que ocorrem exclusiva ou predominantemente durante o sono, de forma intercorrente ou intermitente. São classificadas em três grupos: distúrbios do despertar, parassonias usualmente relacionadas ao sono REM e outras parassonias (Tabela II). De maior interesse no contexto dos distúrbios respiratórios do sono são os distúrbios do despertar. 
Tabela II: Classificação das Parassonias.

- Distúrbios do despertar (a partir de sono não REM)

Despertar confusional

Sonambulismo

Terror noturno

- Parassonias usualmente relacionadas ao sono REM Distúrbio comportamental do sono REM

Paralisia do sono isolada

Pesadelos

- Outras parassonias

Distúrbios dissociativos relacionados com o sono

Enurese do sono

Gemido relacionado ao sono

Síndrome da cabeça explodindo

Alucinações relacionadas com o sono

Distúrbio alimentar relacionado com o sono

Parassonia não especificada

Parassonia associada ao uso de drogas ou substâncias

Parassonia associada à condição médica

Fonte: International Classification of Sleep Disorders - 2nd. ed ${ }^{12}$

As parassonias mais prevalentes são os distúrbios do despertar, ocorrendo mais comumente em crianças e adolescentes, com pico de incidência dos 5 aos 11 anos de idade, persistindo em alguns casos durante a idade adulta. Os episódios tendem a iniciar durante o sono de ondas lentas (fases 3 e 4 do sono não REM) e portanto predominam no primeiro terço da noite. Durante os episódios, o paciente apresenta registro eletrencefalográfico típico de sono superficial (fase I) ou ritmo alfa similar ao da vigília, porém mais lentificado e com pouca reatividade à abertura ocular.

Os distúrbios do despertar compreendem os seguintes quadros: despertar confusional, terror noturno e sonambulismo.

O despertar confusional ocorre principalmente em crianças com menos de 5 anos e geralmente representa um quadro com menor risco de lesões corporais. O paciente apresenta-se nitidamente desorientado, com pensamento lentificado, fala desconexa logo após despertar, podendo manter-se assim por vários minutos. Assim como no quadro de terror noturno e sonambulismo, ocorre amnésia para o evento no dia seguinte.

Durante um episódio de terror noturno, o paciente tipicamente senta na cama, emite gritos estridentes, exibindo evidente ativação autonômica, com taquicardia, taquipnéia, sudorese e midríase. Os episódios não se associam a sonhos vívidos; raramente o paciente se recorda de imagens pouco detalhadas, permitindo a diferenciação com pesadelos. O paciente não se mostra consolável e o episódio tende a terminar espontaneamente.

O sonambulismo pode se manifestar de forma calma ou agitada, com variável nível de complexidade do comportamento motor. Tipicamente ocorre um comportamento automático, como uma tentativa de realizar uma ação com propósito, porém de forma ineficiente. $\mathrm{O}$ paciente pode murmurar ou mesmo emitir sons compreensíveis, mas a comunicação durante o episódio é inviável. A atividade motora pode determinar uma situação de risco para o paciente, que pode tentar sair de casa e mesmo dirigir. $\mathrm{O}$ episódio pode terminar espontaneamente ou após um despertar forçado. Não é incomum a associação com outros tipos de parassonias (overlap syndrome).

Os distúrbios do despertar resultam da dissociação de fenômenos fisiológicos esperados para o sono não REM e a vigília. Tal dissociação implica, por exemplo, na presença de atividade motora típica da vigília, durante estado de sono eletrograficamente determinado. Existem fatores genéticos ${ }^{20,21,22}$ e ambientais relacionados, podendo os ataques serem desencadeados por febre, álcool, privação de sono, stress emocional (Tabela II). Além destes, qualquer distúrbio do sono capaz de induzir à fragmentação do sono, favorecendo, portanto, à dissociação das variáveis fisiológicas, pode potencialmente provocar tais parassonias. Os distúrbios respiratórios do sono ocorrem mais freqüentemente em crianças e adultos que apresentam parassonias $^{23,24}$.Episódios freqüentes de parassonia em pacientes roncadores devem levantar a suspeita de distúrbios respiratórios do sono, devendo ser realizada investigação apropriada com polissonografia. $\mathrm{O}$ tratamento da apnéia do sono muitas vezes tem caráter resolutivo também em relação às parassonias. Surpreendentemente, pacientes com apnéia do sono em início de tratamento com CPAP podem agudamente apresentar episódios de parassonias, associados ao rebote de sono de ondas lentas ${ }^{25}$.

O principal diagnóstico diferencial deve ser feito entre as parassonias e crises epilépticas, o que pode ser clinicamente difícil. As crises epilépticas tendem a ter duração mais limitada e o comportamento motor é mais estereotipado, se constituindo de uma seqüência motora previsível. O paciente pode exibir alterações eletrográficas ictais ou interictais. Em casos selecio- 
Tabela III: Fatores desencadeantes das parassonias.

Privação de sono

Álcool

Stress emocional

Atividade física

Medicações: sedativos hipnóticos, neurolépticos, anti-histamínicos

Gravidez

Distúrbios do sono: apnéia do sono, movimentos periódicos de membros, epilepsia noturna.

nados, a realização de polissonografia com montagem de canais de EEG adicionais e com gravação audiovisual auxilia neste diagnóstico diferencial. $\mathrm{O}$ diagnóstico de casos típicos de parassonia é feito somente através da anamnese.
Como parte da abordagem terapêutica dos distúrbios do despertar, a orientação quanto aos fatores precipitantes e à proteção relacionada ao ambiente (remoção de objetos pérfuro-cortantes, colocação de grades nas janelas) e quanto ao caráter benigno e transitório dos eventos pode ser suficiente. É importante sempre desaconselhar privação de sono e horários irregulares de sono, pois estes podem desencadear os eventos de parassonias. Em casos mais drásticos, seja pela alta frequiência dos episódios, pelo impacto na rotina familiar ou pelo potencial evidente de lesão corporal, o tratamento farmacológico se faz necessário. As drogas de escolha nestas situações são os antidepressivos tricíclicos e benzodiazepínicos. Estes últimos devem ser utilizados com cautela em pacientes roncadores, pelo agravamento da função respiratória durante o sono. Outras opções de tratamento, a partir de relatos de casos, são a trazodona e a paroxetina ${ }^{25,26}$. Em crianças, o tratamento através do despertar antecipatório pode ser efetivo ${ }^{27}$.

Sander HH, Pachito DV, Vianna LS. Sleep disorders associated with Sleep Apnea Syndrome. Medicina (Ribeirão Preto) 2006; 39 (2): 205-211.

ABSTRACT: Many sleep disorders may coexist with apnea/hypopnea syndrome, especially those who are caused by sleep fragmentation. In this review we will focus some of them as insomnia, sleep bruxism and somnambulism.

Insomnia can be referred by patients with apnea. As both disorders can cause severe health problems as car accidents and cardiovascular morbidity and some of the pharmacological treatment of insomnia aggravate apnea, the identification of sleep disordered breathing in patients with insomnia is very important for the correct treatment of both syndromes.

Sleep bruxism can be present in as many as $14 \%$ of patients with apnea. Its severity may be aggravated by apnea and the therapeutic approach must include its treatment.

Somnambulism is one of the most common parasomnias in both children and adults. Patients who frequently snore and presents parasomnias should be evaluated carefully as sleep fragmentation caused by sleep disordered breathing can cause or maintain such sleep problem.

In conclusion, identification of other sleep disorders that occur as epiphenomenona or as consequence of sleep apnea is of extreme importance as both treatment of such disorders can aggravate apnea as its misdiagnosis can interfere with clinic improve those patients.

Keywords: Insomnia. Sleep Bruxism. Somnambulism. Sleep Apnea.

\section{REFERENCIAS}

1 - World Health Organization. Regional Office for Europe. European Center for Environment and Health Bonn Office. WHO Technical Meeting on Sleep and Health-Meeting Report. Bonn Germany: WHO; 2004.
2 - Lichstein KL, Riedel BW, Lester KW, Aguillard. Occult sleep apnea in a recruited sample of older adults with insomnia. $J$ Consult Clin Psychol 1999; 67: 405-10.

3 - Krakow B, Melendrez D, Ferreira E, Clark J, Warner TD. Sisley BN, Sklar D. Prevalence of Insomnia symptoms in patients with sleep-disordered breathing. Chest 2001; 120: 1923-9. 
4 - Chung K-F, Krakow B, Melendrez D, Warner TD, Sisley BN Relationships between insomnia and sleep-disordered breathing. Chest 2003; 123: 310-3.

5 - Smith S, Sullivan K, Hopkins W, Douglas J. Frequency of insomnia report in patients with obstructive sleep apnoea hypopnea syndrome (OSAHS). Sleep Med 2004; 5:449-56.

6 - Krell SB, Kapur VK. Insomnia complaints in patients evaluated for obstructive sleep apnea. Sleep Breath 2005; 9:10410.

7 - American Academy of Sleep. Sleep-related breathing disorders in adults: recommendations for syndrome definition and measurement techniques in clinical research. The Report of an American Academy of Sleep Medicine Task Force. Sleep 1999; 22:667-89.

8 - Zorich FJ, Walsh JK. Evaluation and management of insomnia: an overview. In: Kryger MH, Roth T, Dement C, eds. Principles and practice of sleep medicine, $3^{\text {th }}$. ed. Philadelphia: WB Saunders; 2000. Section 9. p. 615-23.

9 - Sateia MJ, Nowell PD. Insomnia. Lancet 2004;364 (9449):1959-73.

10 - Nancy A. Collop. Can't Sleep? You May Have Sleep Apnea! Chest 2001; 120: 1768-9.

11 - Morin CM. Psychological and behavioral treatments for primary insomnia. In: Kryger MH, Roth T, Dement C, eds. Principles and practice of sleep medicine, $4^{\text {th }}$. ed. Philadelphia: Elsevier Saunders; 2005: Section 9. p.726-37.

12 - American Academy of Sleep Medicine. The International Classification of Sleep Disorders. Westchester, Illinois: American Academy of Sleep Medicine; 2001. p. 182-5.

13 - Kato T, Blanchet PJ, Montplaisir JY, Lavigne GJ. Sleep bruxism and other disorders with orofacial activity during sleep. In: Chokroverty S, Hening WA, Walters AS, eds. Sleep and movement disorders. Philadelphia, Palo Alto: Butterworth Heinemann; 2003. Cap 24, p.273-85.

14 - Ohayon MM, Li KK, Guilleminault C. Risk factors for sleep bruxism in the general population. Chest 2001; 119:53-61.

15 Sjoholm T, Lowe AA, Miyamoto K, Fleetham JA, Ryan CF. Sleep bruxism in patients with sleep-disordered breathing. Arch Oral Biol 2000; 45:889-96
16 - Ayappa I, Rapaport BS, Norman RG, Rapoport DM. Immediate consequences of respiratory events in sleep disordered breathing. Sleep Med 2005; 6:123-30

17 - Phillips BA, Okeson J, Paesani D, Gilmore R. Effects of sleep position on sleep apnea and parafunctional activity. Chest 1986; 90:424-9.

18 - Okeson JP, Phillips BA, Berry DT, Cook YR, Cabelka JF. Nocturnal bruxism events in patients with sleep-disordered breathing and control subjects. J Craniomandib Disord 1991 5:258-64.

19 - Lavigne GJ, Manzini C, Kato T. Sleep bruxism. In: Kryger MH, Roth T, Dement C, eds. Principles and practice of sleep medicine, $4{ }^{\text {th }}$ ed. Philadelphia: Elsevier Saunders; 2005. Chap 79. p. $946-59$

20 - Lecendreux M, Basseti C, Dauvelliers Y, Mayer G, Neidhart E, Tafti M. HLA and genetic susceptibility to sleepwalking. Mol Psychiatry 2003; 8: 114-7.

21 - Hori A, Hirose G. Twin studies on parasomnias. Sleep Res 1995; 24A: 324

22 - Espa F, Dauvilliers Y, Ondze B, Billiard M, Besset A. Arousal reactions in sleepwalking and night terrors in adults: the role of respiratory events. Sleep 2002; $25: 871-5$

23 - Guilleminault C, Palombini L, Pelayo R, Chervin RD. Sleepwalking and sleep terrors in prepuberal children: what triggers them? Pediatrics 2003, 111: 17-25

24 - Millman RP, Kipp GR, Carskadon MA. Sleepwalking precipitated by treatment of sleep apnea with nasal CPAP. Chest $1991 ; 99: 750-1$

25 - Lillywhite R, Wilson SJ, Nutt DJ. Successful treatment of night terrors and somnambulism with paroxetine. Br J Psychiatry $1994 ; 164: 551-4$

26 - Balon R. Sleep terror disorder and insomnia with trazodone: a case report. Ann Clin Psychiatry 1994; 6:161-3

27 - Mahowald MW, Schenck C. NREM parasomnias. Neurol Clin 1996; 14:697-720 